\title{
EL POTENCIAL HUMANO Y LOS ESTÍMULOS ORGANIZACIONALES
}

\author{
Pedro L. Tito Huamaní * \\ E-mail: ptitoh@unmsm.edu.pe \\ Juan Castillo Maza*** \\ E-mail: jcastillom@unmsm.edu.pe
}

\section{RESUMEN}

Los resultados de la presente investigación revelan las estrategias que se deben utilizar para motivar al trabajador promedio en instituciones asistenciales del Sector Salud. Éstas se relacionan con la satisfacción de necesidades de capacitación, promoción y ascensos, y reconocimiento institucional al trabajo desarrollado. En este orden revelado por las encuestas aplicadas a los trabajadores del Instituto Especializado Materno Perinatal (IEMP), los estímulos ligados a aspectos económicos y pecuniarios ocupan el tercer grupo de preferencias después de la mejora de condiciones laborales, vía equipamiento permanente y materiales adecuados para su trabajo. Tales resultados son inversos a lo propuesto por Abraham Maslow, en su famosa teoría de la jerarquía de necesidades.

Los resultados también revelan que los trabajadores del Sector Salud -vía investigación en el IEMPdemandan una dirección institucional democrática y participativa, que les permita tomar decisiones en actividades inherentes a su función y aportar constructivamente en el desarrollo institucional. Ello implica un cambio de paradigmas, de los directivos y jefes intermedios, quienes deben aprender a confiar y delegar las funciones en sus colaboradores.

Palabras clave: Motivación, Colaborador, Gestión del Potencial Humano.

\section{ABSTRACT}

The results of the present investigation reveal the strategies that should be used to motivate the worker average in assistance institutions of the Sector Health. These they are related with the satisfaction of training necessities, promotion and ascents, and institutional recognition to the developed work. This order revealed by the surveys applied the workers of the Perinatal Maternal Specialized Institute (IEMP), the bound stimuli to economic and pecuniary aspects occupy the third group of preferences after the improvement of labor conditions, via permanent equipment and appropriate materials for their work. Such results are inverse to that proposed by Abraham Maslow, in their famous theory of the hierarchy of necessities.

* Licenciado y Magíster en Administración UNMSM. Docente de la Facultad de Ciencias Administrativas UNMSM, especialista en Comportamiento Organizacional y Gestión del Potencial Humano.

*** Licenciado en Administración por la Universidad Nacional de Trujillo; Magíster en Economía con mención en Métodos Cuantitativos y Doctor en Ciencias Contables y Empresariales por la Universidad Nacional Mayor de San Marcos; Profesor Principal de la UNMSM; Encargado de la Dirección de la Escuela Académico Profesional de Administración; Director del Comité Editorial de 
The results also reveal that the workers of the Sector Health - via investigation in the IEMP - they demand a democratic institutional address that allows them to make decisions in inherent activities to their function and to contribute constructively in the institutional development. It implies a change of paradigms, of the directive and intermediate bosses who should learn how to trust and to delegate the functions in their collaborators.

Key words: Motivation, Collaborator, Administration of the Human Potential.

\section{INTRODUCCION}

La nueva conceptualización del valor de las personas para las organizaciones se inicia con los planteamientos efectuados por las diferentes escuelas que estudian la Gestión de las Personas, desde el punto de vista las ciencias del comportamiento. En ésta se abandona la idea de que la persona, en sí misma, es un recurso, es decir un elemento que provee de fuerza de trabajo a la organización tal y como se concebía desde los tiempos de los autores clásicos de la Administración, para pasar a la consideración de la persona como un elemento dinámico que posee competencias, talentos y recursos; vale decir, posee conocimientos y capacidades, habilidades y destrezas, y actitudes.

Tales recursos, fuente de ventaja competitiva, deben ser gestionados y potenciados convenientemente para conseguir su movilización en torno a los objetivos generales que las organizaciones se proponen lograr. Para ello, las estrategias que se diseñen y ejecuten deben compatibilizar el desarrollo organizacional con el desarrollo integral de la persona, vale decir crecimiento personal y profesional.

Así, en el tema de la Gestión del Potencial humano, de una concepción micro organizacional, estático y transaccional, preocupada a mantener el status quo y los sistemas de control interno en los puestos de trabajo, propios de la mayoría de organizaciones en nuestro medio; pasamos a una función eminentemente directiva, organización integral, dinámica y en constante transformación, vinculada al desarrollo y a la flexibilización de los sistemas de trabajo y al cambio organizacional, propio de organizaciones de vanguardia, con el agregado de que los resultados tienden a ser intangibles, difícilmente medibles, pero que por su dimensión el cambio se convierte en una función eminentemente estratégica para la organización.

Precisamente el presente estudio se orienta a demostrar que una gestión organizacional de las personas, basada en la valoración individual y colectiva del colaborador, a partir de la aplicación de estrategias motivacionales acordes a sus necesidades, será significativamente superior que aquella gestión que se circunscribe a la preocupación y búsqueda permanente de alcanzar solo los logros organizacionales, subvaluando y menospreciando los aportes de sus trabajadores.

Esta premisa, que bien puede ser la consecuencia de un razonamiento lógico fue validada en el trabajo empírico con la investigación realizada tanto a los directivos y trabajadores como a las pacientes "clientes" del Instituto Especializado Materno Perinatal (IEMP), entidad pública del Sector Salud, líder en el campo de la Ginecoobstetricia y Neonatología.

\section{PLANTEAMIENTO Y JUSTIFICACIÓN DEL PROBLEMA}

Existen en el Instituto Especializado Materno Perinatal -Ex Maternidad de Lima- diversas unidades que realizan actividades principales y de apoyo a través de sus trabajadores, hoy concebidos como colaboradores ${ }^{1}$. Como es sabido, el éxito de las organizaciones a largo plazo depende de la gestión de tales colaboradores, porque son ellos los que producen la buena o mala calidad de los servicios que ofrece la institución. En este sentido, la gestión del potencial humano en el IEMP involucra a la Alta Dirección, a los niveles de decisión intermedia y a los niveles operativos que constituyen el entorno especifico de la Oficina de Gestión de Recursos Humanos. 
Por otro lado, al igual que todas las instituciones antiguas, el IEMP ha desarrollado un conjunto de valores a partir de las costumbres, creencias y ritos que en el tiempo se fueron cultivando; también aplica políticas y estrategias del sector que muchas veces cambian con los gobiernos de turno, condicionando la calidad de gestión de sus colaboradores.

Algunos aspectos sintomáticos que identifican la problemática de la gestión del Talento Humano en el IEMP son: la indisciplina de un sector reducido de profesionales y trabajadores asistenciales que generan conflictos y enervan el clima laboral, fricciones con sus demás compañeros de trabajo y miembros del Equipo de Gestión, baja productividad reflejada en los costos operativos elevados que encarecen la atención a los pacientes, algunos servicios catalogados como de "mala calidad" que brindan a las pacientes "clientes", quejas de pacientes sobre el descuido e insensibilidad de trabajadores tanto profesionales como técnicos, incumplimiento en algunos de sus metas de atención programadas (cesáreas, intervenciones quirúrgicas, legrados, curetajes, consultorios, etc.), pacientes que reingresan al instituto después de su alta por dolores o malas prácticas médicas, existencia aproximada del 5\% de trabajadores marginales con problemas de drogadicción y alcoholismo que influyen en su desempeño laboral; entre otros.

Tales situaciones descritas, comunes en centros asistenciales públicos, deben ser corregidas por la Alta Dirección de la institución a través de prácticas gerenciales de liderazgo y gestión participativa, de sensibilización y motivación permanente a los colaboradores, diseño y ejecución de programas de capacitación laboral para que mejoren sus habilidades y destrezas e incorporen nuevos conocimientos, entre otros.

\section{Justificación Teórica Metodológica y Práctica}

Una ampulosa teoría acerca del comportamiento humano sustenta que existe una diversidad de estímulos que se pueden utilizar en el trabajador para obtener mayores y mejores rendimientos. Ello significa que tales rendimientos se pueden conseguir utilizando estímulos sociales y psicológicos, económicos, materiales, etc.; mejor dicho, estos estímulos irán variando de trabajador en trabajador, en función del nivel socioeconómico, formación y expectativas que tiene cada uno.

La investigación propuesta busca -mediante la aplicación de conceptos y teorías de motivación, liderazgo, trabajo en equipo y empowerment, dentro del ámbito de Gestión del Potencial Humano ${ }^{2}$ - encontrar explicaciones para elevar el nivel de productividad y la calidad de servicios que se ofrece en el IEMP. Para ello se hace necesario desarrollar un marco teórico y conceptual revisando la amplia bibliografía existente, contrastando las diversas corrientes y posiciones, y a partir de ella comprobar su validez en una organización pública encargada de brindar salud a la sociedad peruana.

Los alcances de la investigación muestran la importancia que tiene para el IEMP la aplicación de un modelo de gestión que propenda a convertirla en una institución líder del sector salud, no sólo como centro de referencia nacional en gineco-obstetricia y neonatología, sino también como referente institucional que hace uso de un modelo de gestión organizacional.

La importancia práctica de esta investigación también se justifica por cuanto, siendo el IEMP un centro altamente especializado de cuarto nivel, para conservar tal estatus requiere identificación y compromiso de sus colaboradores con sus propósitos, y una preparación constante para su mejor desempeño laboral. Tal escenario antes descrito solo se puede lograr con una administración profesional que tome en cuenta los aportes de sus colaboradores.

Otro destacable es la observación de que las demás instituciones públicas tales como institutos y hospitales generales del sector salud, se caracterizan por tener comportamientos organizacionales muy similares. De modo que las conclusiones obtenidas en la presente investigación pueden servir para inferir a las realidades de tales instituciones.

\section{Limitaciones de la investigación}

La presente investigación presenta limitaciones desde el punto de vista geográfico, en el sentido que, en el mejor de los casos, sus resultados y conclusiones solo se pueden inferir a realidades de hospitales generales e institutos del sector salud ubicados en la capital, lo cual 
implica que para validar tales resultados y conclusiones en instituciones asistenciales ubicadas en ciudades del interior del país deberán complementarse con otras investigaciones que recojan las culturas y realidades de tales centros asistenciales.

De la misma forma, las conclusiones y recomendaciones de la presente investigación no podrán inferirse como válidos sino se complementan con otras investigaciones en los institutos especializados y clínicas, que por ser privadas tienen una concepción y cultura organizacional eminentemente empresarial, lo cual dista mucho de las instituciones asistenciales del sector público, que entre otros se caracterizan por financiar sus programas de desarrollo con recursos provenientes del erario nacional y, en proporciones reducidas, con donaciones y recursos de la cooperación internacional.

\section{Objetivos de la Investigación}

Identificar y proponer estrategias gerenciales y administrativas que permitan mejorar la gestión del potencial humano del Instituto Especializado Materno Perinatal (IEMP). Para ello, es vital analizar e interpretar los factores que condicionan dicha gestión, identificando y describiendo los estilos de gestión que aplican los Directivos y Ejecutivos de la institución, y, de ser posible, formular escenarios alternativos que configuren un modelo de gestión efectivo para este componente importante en el IEMP.

\section{Hipótesis de la Investigación}

La aplicación de estrategias de gestión del potencial humano que promueven una dirección participativa, la capacitación permanente y una retribución salarial sobre la base del reconocimiento de su eficiencia, mejorará el nivel de Productividad y la Calidad de los servicios que ofrecen a sus pacientes el IEMP.

Las siguientes son las hipótesis auxiliares:

a) La capacitación laboral permanente permite mejorar las habilidades y destrezas e incorporar nuevos conocimientos en los trabajadores.

b) El reconocimiento institucional al esfuerzo que realiza el trabajador, traducido en la promoción, ascensos y facilidades de equipos y materiales, son factores motivadores para el trabajador del IEMP.
Variables Independientes

1. Reconocimiento institucional al trabajador

2. Capacitación laboral

3. Estímulos económicos y materiales

Variables Dependientes

- Satisfacción del trabajador en la prestación de un servicio de calidad.

\section{ASPECTOS METODOLÓGICOS}

La investigación del estudio propuesto se realizó utilizando el Método Científico, vale decir, cuando se tienen los elementos que determinan lo que se va a analizar es preciso establecer métodos y procedimientos de investigación que permitan solucionar problemas institucionales y/o establecer mecanismos que científicamente sean válidos en la solución de problemas similares en otras instituciones.

Al respecto, como existen numerosos procedimientos o técnicas para obtener información acerca del problema e hipótesis, en la presente investigación se ha procedido a utilizar una combinación de métodos, entre los cuales destacan: recopilación y análisis documental, elaboración y aplicación de cuestionarios ad hoc, análisis de los resultados haciendo uso de procedimientos estadísticos y programas informáticos, complementados con entrevistas, entre otros.

\section{Población y Muestra}

El universo de la población investigada involucró a todos los miembros del IEMP que suman 1,460 trabajadores, teniendo como referencia el rol que cumplen actualmente, ya sea por las funciones asignadas o por el nivel de formación que ostentan. En base a la información obtenida se ha considerado una población muestral equivalente al $13 \%$ del total de los miembros del IEMP, partiendo de la premisa de que dicha muestra es por conveniencia y criterial en función del problema a investigar, distribuidos de la siguiente forma:

\begin{tabular}{lc}
\hline $\begin{array}{c}\text { Trabajadores según grupo } \\
\text { ocupacional }\end{array}$ & Muestra \\
\hline Médicos & 32 \\
Obstetrices & 23 \\
Enfermeras & 25 \\
Técnico en Enfermería & 36 \\
Otros trabajadores asistenciales & 27 \\
Trabajadores Administrativos & 43 \\
MUESTRA TOTAL & $\mathbf{1 8 6}$ \\
\hline
\end{tabular}


El trabajo de campo se realizó utilizando el método de muestreo aleatorio simple, esto es, todos los elementos de la población tienen las mismas posibilidades de ser elegidos, eliminando con ello la carga subjetiva del investigador y las posibilidades de sesgar el estudio.

De la misma forma, para contrastar y validar la información obtenida por el lado de los miembros del IEMP (oferta), se ha procedido aplicar una encuesta ad hoc a las pacientes "clientes" (demanda), en forma aleatoria tanto en Consultorios Externos como en los servicios de Hospitalización.

\section{Diseño y Aplicación de Cuestionarios}

El cuestionario aplicativo estableció provisionalmente las consecuencias lógicas de los objetivos e hipótesis planteados, que aunados a la razonable experiencia acumulada, sirvieron para contrastar los mismos.

Tales cuestionarios fueron elaborados cuidadosamente y se comprobó su operatividad a través de una prueba piloto que fue desarrollada por un equipo de estudiantes universitarios, previamente seleccionados y capacitados para tal fin. Se tuvo especial cuidado para garantizar que el encuestado conteste libre y voluntariamente, asegurándonos de anotar exactamente sus respuestas.

Para corroborar o ahondar en las informaciones fue necesario complementar con las entrevistas, que se realizaron principalmente a los trabajadores que tienen responsabilidad sobre grupos de colaboradores. De la misma forma fue necesario entrevistar a las pacientes del instituto.

\section{Procesamiento de la Información}

Para el procesamiento de la información recabada a través de las encuestas ad-hoc, fue necesario utilizar diversos tipos de softwares destinados a medir variables cualitativas como el systat, el SPSS, y para las variables cuantitativas se utilizó el statgraphic, entre otros. De la misma forma para la preparación y presentación del informe final fue necesario utilizar los diversos paquetes aplicativos de Microsoft Office.

\section{RESULTADOS DE LA INVESTIGACIÓN}

\section{Características generales de los colaboradores encuestados (Oferta)}

El $77 \%$ son trabajadores que realizan funciones asistenciales, en tanto que el $23 \%$ realiza funciones administrativas. De acuerdo a las estadísticas del Ministerio de Salud, la distribución de trabajadores del IEMP es concordante con los porcentajes nacionales en hospitales generales.

El Gráfico 1 muestra la clasificación de los trabajadores en función a su formación profesional, que también es concordante con los promedios de los hospitales generales. Sin embargo, las excepciones están dadas por el alto número de Obstetrices y por los Médicos GinecoObstetras a nivel de subespecialidades médicas. Tales variaciones se explican porque el IEMP oferta las dos especialidades (Gineco-obstetricia y Neonatología) como principales y los servicios relacionados a ellas, donde las participaciones de los mencionados profesionales es determinante. En relación a los otros profesionales asistenciales están considerados Odontólogos, Tecnólogos Médicos, Químicos, Farmacéuticos, Psicólogos, Asistentas Sociales y Nutricionistas, y los técnicos de mando medio como Técnicos de Laboratorio, Técnicos de Farmacia, Técnicos de Nutrición, Técnicos de Estadística, Auxiliares de Enfermería, entre otros, que como grupo ocupacional no son representativos.

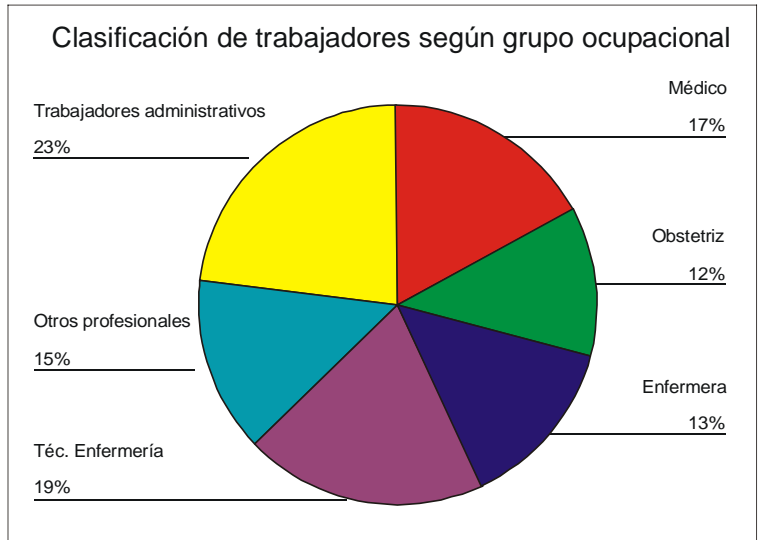

Gráfico 1. Trabajadores según grupo ocupacional.

Tomando en cuenta la edad de su colaboradores (Gráfico 2), el IEMP es una institución madura, dado que la mitad de ellos son mayores de 36 años. 
Del total de los trabajadores el $74 \%$ son mujeres, mientras que el $26 \%$ son hombres; en tanto que, desde el punto de vista de su relación contractual, el 85\% son nombrados, por tanto gozan de estabilidad laboral y los demás beneficios que la legislación laboral lo estipula; $3.2 \%$ son contratados con recursos provenientes del Tesoro Público y el $12 \%$ restante son personal contratado con recursos directamente recaudados por la institución, bajo la modalidad de Servicios No Personales (SNP). Los trabajadores contratados por tesoro público en la práctica son cuasi- nombrados, por cuanto gozan de los mismos beneficios que los nombrados, solo que a diferencia del primer grupo que tiene contrato a plazo indefinido, éstos tienen contratos a plazo fijo. Los contratados por servicios no personales, por la temporalidad de su relación contractual (para una función específica y por un tiempo específico), que en la práctica se convierte en permanente, no tienen derecho a los beneficios que el Estado otorga (vacaciones, gratificaciones, acumulación por tiempo de servicios, descansos, licencias, permisos, etc). Tampoco gozan de los beneficios que la institución otorga (uniforme, bonos por alimentos, incentivos por productividad, bonificación por guardia hospitalaria, vales por movilidad, cambios de turno, entre otros). Dado que son vulnerables, su permanencia en la institución es incierta.

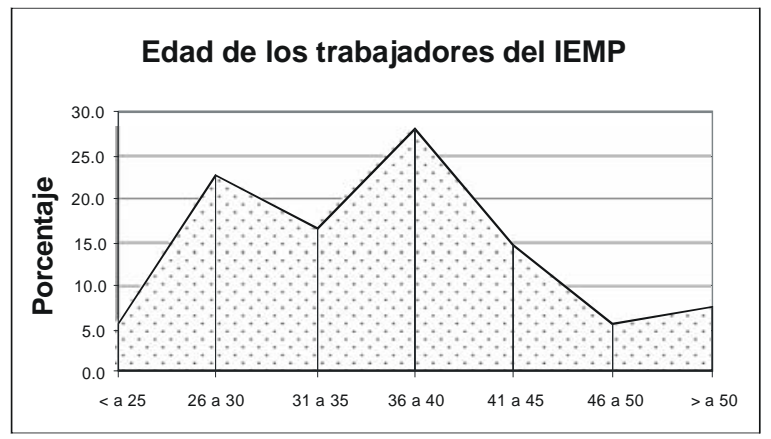

Gráfico 2. Rango de edad.

\section{Características generales de pacientes encuestadas (Demanda)}

De acuerdo a los resultados obtenidos por el lado de la demanda, se tiene que un $18 \%$ de los encuestados reside en el distrito de San Juan de Lurigancho, un $12 \%$ dice residir en el Cercado de Lima, un $10 \%$ para aquellos que residen en Villa María del Triunfo, El Agustino y Santa Anita y un restante $40 \%$ para aquellos que residen en los demás distritos de la ciudad de Lima; de modo que la mayor incidencia de visita a este hospital es la que proviene del distrito de San Juan de Lurigancho. A pesar de la gran distancia que existe desde este distrito hasta la institución, las entrevistadas optan por atenderse en el IEMP por ser la institución más apropiada y especializada en los servicios que ofrece.

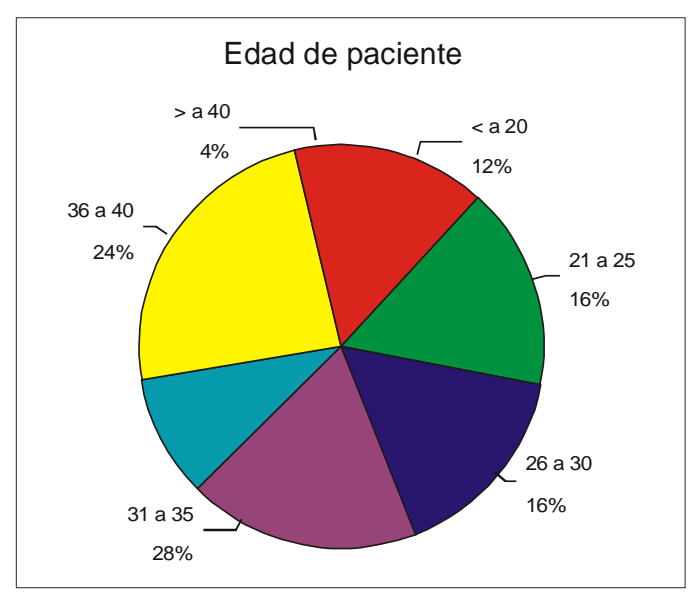

Gráfico 3. Distribución de pacientes según edad.

Con respecto a las edades (Gráfico 3) que ostentan las pacientes que visitan frecuentemente esta institución de salud, casi una tercera parte de la muestra se encuentra entre los 31 y 35 años, mientras que un $24 \%$ oscila entre los 36 y 40 años de edad. El porcentaje restante se reparte entre edades que van desde menos de 20 hasta 30 años de vida.

Cabe resaltar que más del 50\% de las entrevistadas que se atienden en esta institución, según la muestra, tienen edades que oscilan entre 31 y 40 años, entendiéndose que este intervalo corresponde a la etapa de gestación por la que atraviesan la mayor parte de las mujeres en edad fértil.

En lo que se refiere al tipo de atención recibida por parte de las pacientes durante las visitas a la Maternidad de Lima, el $62 \%$ de las encuestadas manifestaron haberse atendido ambulatoriamente. Las que sí recibieron hospitalización representan el 34\% de la muestra consultada y un $4 \%$, manifestaron que fueron atendidas por emergencia.

\section{Nivel de percepción de los colaboradores}

El Cuadro 1 revela las acciones que deben desarrollar las instituciones del Sector Salud para tener trabajadores satisfechos y productivos. Las 
Cuadro 1. Acciones que todo centro asistencial del Ministerio de Salud debe ejecutar para motivar a sus "colaboradores" -Primera Mención-

\begin{tabular}{|c|c|c|c|}
\hline Válidos & & Válido (\%) & Acumulado (\%) \\
\hline & Capacitar periódicamente & 42.5 & 42.5 \\
\hline & Promover el ascenso del personal & 21.5 & 64.0 \\
\hline & Facilitar materiales para su trabajo & 10.8 & 74.7 \\
\hline & Adquirir equipos modernos & 5.4 & 80.1 \\
\hline & Definir claramente las funciones personales & 4.3 & 84.4 \\
\hline & Mejorar los "bonos" por alimentos & 3.2 & 87.6 \\
\hline & Incrementar los "incentivos por productividad" & 2.7 & 90.3 \\
\hline & Financiar estudios de especialización en el extranjero & 2.7 & 93.0 \\
\hline & Facilitar su uniforme y equipos personales de trabajo & 2.2 & 95.2 \\
\hline & Reconocer públicamente su esfuerzo & 1.6 & 96.8 \\
\hline & Otras acciones & 3.2 & 100.0 \\
\hline & Total & 100.0 & \\
\hline
\end{tabular}

más importantes se relacionan con el nivel de satisfacción de necesidades secundarias (capacitación, y promoción y ascensos), seguida de aspectos ligados a su área de trabajo (equipos modernos, facilidad de materiales). Paradójicamente, las motivaciones de orden monetario y material (incrementar los incentivos por productividad y mejorar los bonos por alimentos), ocupan posiciones de tercera prioridad. Esta tendencia fue corroborada posteriormente cuando se procesaron todas las menciones.

Sin embargo cuando se preguntó acerca de los beneficios recibidos directamente por los entrevistados en los últimos doce meses, el 20.4\% consideraron al trato de sus jefes como beneficio, en tanto que el $11.8 \%$ catalogan así al uniforme y equipos de trabajo que recibieron. Las siguientes acciones identificadas por los entrevistados se relacionan a aspectos sociales y pecuniarios, así el haber sido capacitado ocupa el tercer lugar con un $10.2 \%$, en tanto que la mejora de los bonos por alimentos y el incremento de los incentivos económicos ocupan las subsiguientes posiciones con $3.8 \%$ y $3.2 \%$, respectivamente. En este orden de ideas, un $41.9 \%$ de los entrevistados manifestaron que no recibieron ningún beneficio de la institución en los últimos doce meses. Si correlacionamos con el total de trabajadores de la institución, serían aproximadamente 603 personas los que se encuentran en esta situación, cantidad significativa que de no tomarse alguna medida inmediata, puede conducir a una progresiva desmotivación.

Por otro lado, el nivel de percepción que tienen de sus jefes inmediatos superiores (Gráfico 4), es favorable. Frente a la pregunta espe- cífica del trato que reciben de sus superiores, el $75.2 \%$ manifestó que es muy bueno; en tanto que solo el $24.8 \%$ mostró su insatisfacción $(21 \%$ dicen que es regular y $3.8 \%$ argumentan que es malo). Tales resultados son congruentes con el nivel de percepción que tienen acerca del clima laboral reinante al momento de realizar el trabajo de campo. Así, el $68.3 \%$ de ellos catalogaron que el ambiente laboral que reina en la institución es bueno ( $7 \%$ muy bueno y $61.3 \%$ bueno). Sin embargo, al igual que el clima geográfico, el ambiente laboral puede alterarse de un momento a otro como en efecto ocurrió en los días posteriores a la aplicación de la presente encuesta, por los hechos fortuitos de contaminación con sangre $\mathrm{VIH}$ positivo a tres recién nacidos y que fueron de conocimiento de la opinión pública en general.

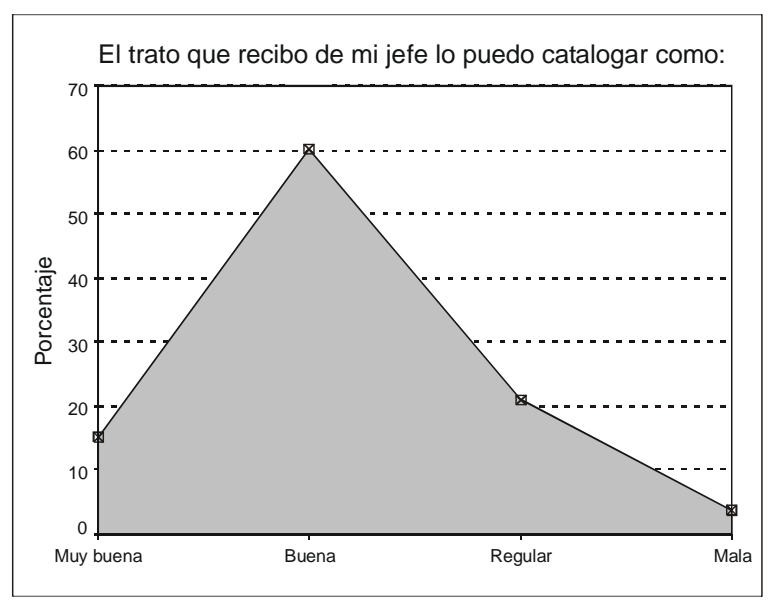

Gráfico 4. Calificación.

En relación al nivel de empoderamiento del que deben gozar los colaboradores del IEMP, los resultados de la investigación son concluyentes 


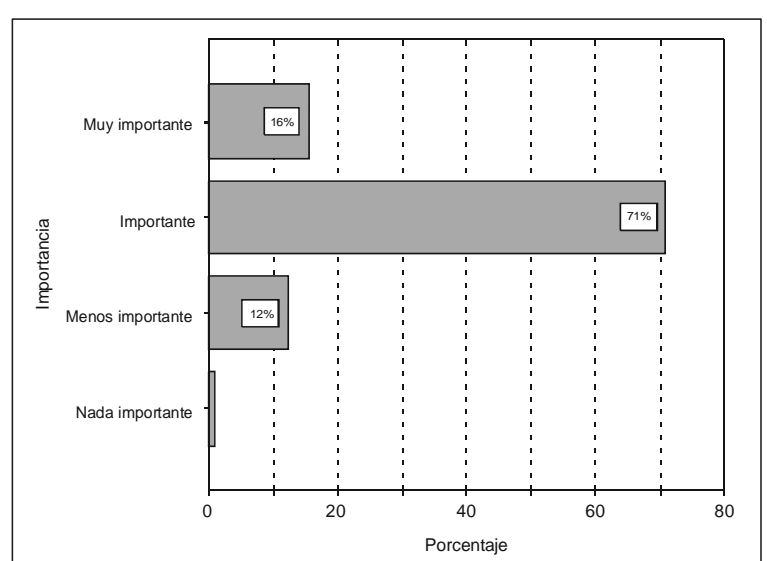

Gráfico 5. Autonomía del personal para tomar decisiones.

a favor de su implantación. Así, de acuerdo con el Gráfico 5, el 87\% de los encuestados consideran que es muy importante/importante gozar de autonomía para tomar decisiones en actividades inherentes a sus funciones, frente al $13 \%$ de aquellos que consideran que la autonomía es menos/ nada importante. Tales resultados son concordantes con el $87.1 \%$ de los encuestados que reclaman a sus jefes para que deleguen las funciones, frente al $12.9 \%$ de ellos que consideran como irrelevante el delegar las funciones en los colaboradores. Llevar a la práctica esta aspiración loable de los trabajadores del Sector Salud, que en el mundo del management es una tendencia en franco crecimiento, debe significar la ruptura del paradigma vigente que descansa sobre bases de mantenimiento del status quo. La delegación de funciones es compatible con gozar de autonomía para tomar decisiones. Tanto el primero como el segundo son sinónimos de confianza, responsabilidad, seriedad y profesionalidad en las funciones encomendadas, pero también lealtad y fidelidad al superior que faculta.

Los incentivos económicos adicionales a sus haberes que actualmente reciben, merced a las $\mathrm{RM} \mathrm{N}^{\circ}$ 827-2004/MINSA, 717-2004/MINSA y 8252003-SA/DM, por conceptos de productividad y asignación extraordinaria, por trabajo asistencial a nivel del sector, así como los incentivos económicos por alimentos a nivel institucional, también fueron motivos de estudio (Gráfico 6). Tales incentivos alcanzan a todos los trabajadores, incluso a aquellos que se benefician con el incentivo por guardia hospitalaria, salvo los contratados por Servicios No Personales (SNP).

Un trabajador que realice el total de días autorizados puede llegar a obtener por este concepto la suma de 600 soles mensuales (20 días

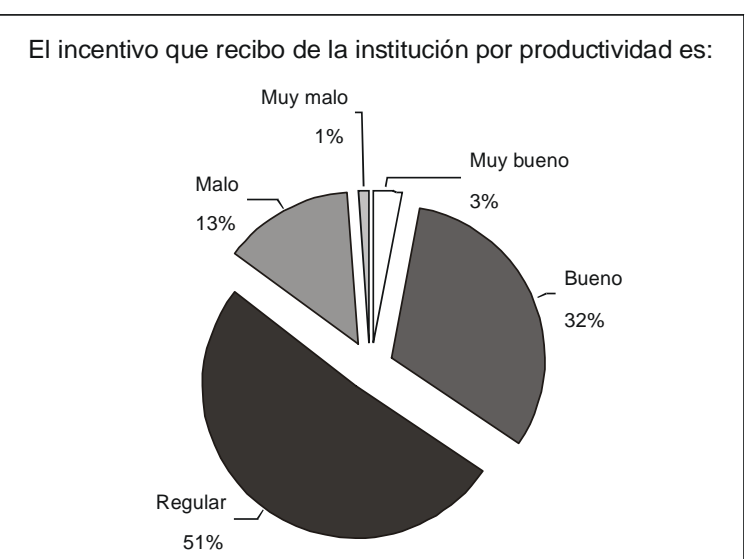

Gráfico 6. Incentivo económico por PRODUCTIVIDAD.

x S/.30 por día). Al respecto, solo el 30.7\% considera este incentivo como factor motivante $(2.7 \%$ muy bueno y $28 \%$ bueno); en tanto que $57.5 \%$ considera lo contrario ( $44.6 \%$ regular, $11.8 \%$ malo y $1.1 \%$ muy malo). El $11.8 \%$ de los trabajadores manifestaron no recibir ninguno de los beneficios económicos, dada su condición laboral de ser contratados por SNP. Dicha posición es concordante con los beneficios que proporciona la institución por concepto de bono por alimentos, que asciende a la suma de $\mathrm{S} / .230$ mensuales. Al respecto, sólo el $19.9 \%$ considera que es un buen incentivo, en tanto que el $55.4 \%$ considera que es regular; mientras que el $12.3 \%$ de los trabajadores catalogan como malo $(11.8 \%)$ y muy malo $(0.5 \%)$. De la misma forma el $11.8 \%$ opinó no beneficiarse.

El Gráfico 7 resume el nivel de percepción que tienen los colaboradores de las condiciones laborales del IEMP, ya sea para favorecer o desfavorecer su desarrollo personal y profesional.

A pesar de los problemas y dificultades por los que suelen pasar las instituciones asistenciales

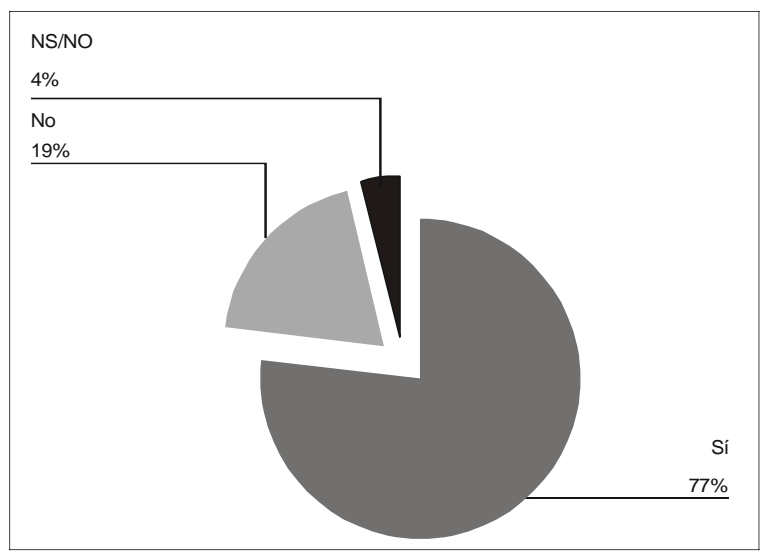

Gráfico 7. Nivel de percepción de las condiciones laborales, que favorecen su desarrollo personal. 
públicas, como es el caso del Instituto Especializado Materno Perinatal, el $76.9 \%$ de los trabajadores sostiene que dichas condiciones sí favorecen a su desarrollo personal y profesional; en tanto que una minoría que representa $19.4 \%$ sostiene lo contrario. Esta percepción favorable de los trabajadores para continuar apostando por el IEMP se explica por su tradición, el tipo de servicio que brinda que es único en su género, la experiencia acumulada, ser considerado referente nacional e internacional en el campo de la Gineco- Obstetricia y Neonatología, entre otros.

Las razones de los que se mostraron contarios a tener una opinión favorable, se reflejan en el Cuadro 2. Así, el $28.9 \%$ de los insatisfechos creen que la institución no capacita a sus trabajadores, mientras que el $15.8 \%$ manifiestan que hay mucha restricción en la institución por la rigidez de las normas. Así mismo, el $13.2 \%$ de los insatisfechos arguyen que no se valoran las iniciativas personales, en tanto que el $10.5 \%$ opinan que la institución no facilita materiales para realizar su trabajo.

La indiferencia de los directivos y jefes a sus colaboradores, y la carencia de facilidades para que los trabajadores tomen decisiones por iniciativa propia, ocupan el quinto y sexto lugar, respectivamente. En este último puesto, empatan la carencia de infraestructura adecuada, condiciones económicas inadecuadas, beneficios de la institución solo a argollas, el no involucrar a los contratados por SNP y la política de «ninguneo» de los jefes, con el 7.9\%

En esta parte, es importante recordar lo mencionado por el $41.9 \%$ de los entrevistados, que argumentaron no haber recibido ningún beneficio en los últimos 12 meses. De modo que este $19.4 \%$ de los insatisfechos posiblemente estén incluidos en el grupo de los que no fueron beneficiados últimamente.

Otro tema de investigación estuvo referido a conocer el procedimiento que adoptan los jefes de unidades y áreas del IEMP frente al surgimiento de los problemas, tal como podemos ver en el Cuadro 3. Tales resultados muestran que en la institución coexisten 3 estilos jefaturales, el primero que se caracteriza por ser autoritario y dominante $(42.5 \%)$, el segundo que es indiferente a la realidad de la unidad o área de trabajo (16.7\%), y el tercero que escucha a los trabajadores y toma decisiones por consenso (40.9\%).

Cuadro 2. Razones por las cuales considera que las condiciones laborales del IEMP no favorecen a su desarrollo personal y profesional.

\begin{tabular}{lrc}
\hline & Válido (\%) & Acumulado (\%) \\
\hline No fomentan la capacitación permanente & 28.9 & 28.9 \\
Las normas son rígidas, hay mucha restricción & 15.8 & 44.7 \\
No apoyan las iniciativas individuales & 13.2 & 57.9 \\
No facilitan materiales para el trabajo & 10.5 & 68.4 \\
Indiferencia de los Directivos al personal & 10.5 & 78.9 \\
No permiten tomar decisiones fuera del esquema & 7.9 & 86.8 \\
Carencia de infraestructura adecuada & 7.9 & 94.7 \\
Las condiciones económicas son inadecuadas & 7.9 & 102.6 \\
Benefician solo a argollas & 7.9 & 110.5 \\
Los beneficios no alcanzan a los contratados SNP & 7.9 & 118.4 \\
Política de «ninguneo» de los jefes & 5.3 & 123.7 \\
Otras razones & 13.2 & 136.9 \\
Total (") & $>=100.0$ &
\end{tabular}

$\left({ }^{*}\right)$ : Son mayores al $100 \%$, porque se consideran todas las opiniones de trabajadores que no se sienten favorecidos con las condiciones laborales del IEMP

Cuadro 3. Procedimientos adoptados por el jefe ante el surgimiento de problemas.

\begin{tabular}{lcccc}
\hline & Frecuencia & Porcentaje & Porcentaje válido & Porcentaje acumulado \\
\hline Convoca y reprocha en público & 15 & 8.1 & 8.1 & 8.1 \\
Toma la decisión y ordena las funciones & 64 & 34.4 & 34.4 & 42.2 \\
No le interesa & 31 & 16.7 & 16.7 & 59.1 \\
Se preocupa y evita profundizar & 32 & 17.2 & 17.2 & 76.3 \\
Analiza y decide por consenso & 44 & 23.7 & 23.7 & 100 \\
Total & $\mathbf{1 8 6}$ & $\mathbf{1 0 0}$ & $\mathbf{1 0 0}$ & \\
\hline
\end{tabular}


Ahora bien, analizando a profundidad esta tendencia a nivel de los diversos grupos ocupacionales, tal como se muestra en el Cuadro 4, vemos que es en las Obstetrices y Trabajadores Administrativos donde se acentúa el estilo autoritario y dominante; en tanto que a nivel de los Médicos y Enfermeras se acentúa el estilo democrático y participativo.

\section{ANÁLISIS E INTERPRETACIÓN CRUZANDO MÁS DE UNA VARIABLE}

En el interés de obtener resultados significativos, en esta parte se ha procedido a cruzar variables que guardan relación o muestran tendencias relevantes para los propósitos de la presente investigación. Para ello fue necesario recurrir a los conceptos y procedimientos estadísticos que, como bien sabemos, aclaran mejor los resultados para su posterior análisis e interpretación.

Por ello, a fin de contrastar los supuestos se utilizó la distribución chi-cuadrado en razón de que los datos disponibles para el análisis están distribuidos en frecuencias absolutas o frecuencias observadas. El estadístico chi-cuadrado es el más adecuado para esta investigación, por cuanto las variables son cualitativas y están definidas en escala de medición ordinal y nominal.

Procedimientos adoptados por el jefe ante el surgimiento de problemas frente a la clasificación de trabajadores según el grupo ocupacional.

\section{Hipótesis C:}

$\mathrm{H}_{\mathbf{0}}$ : Los procedimientos adoptados por el jefe ante el surgimiento de problemas es independiente de la opinión que tienen al respecto los trabajadores, según el grupo ocupacional al que pertenecen.

$\mathbf{H}_{1}$ : Los procedimientos adoptados por el jefe ante el surgimiento de problemas influyen positivamente en la opinión que tienen al respecto los trabajadores, según el grupo ocupacional al que pertenecen.

Los datos han sido clasificados en una tabla de contingencia para probar la hipótesis de independencia de criterios. Se ha considerado un nivel de significancia $\mathbf{a}=0.05$ y 1 grados de libertad, cuyo valor tabular es de $\mathbf{c}_{(0.05,1)}^{2}=3.8416$, que luego será comparado con un chi-cuadrado experimental para la aceptación o rechazo de la hipótesis nula.

Cuadro 4. Procedimientos adoptados por el jefe ante el surgimiento de problemas vs. Clasificación de trabajadores según grupo ocupacional.

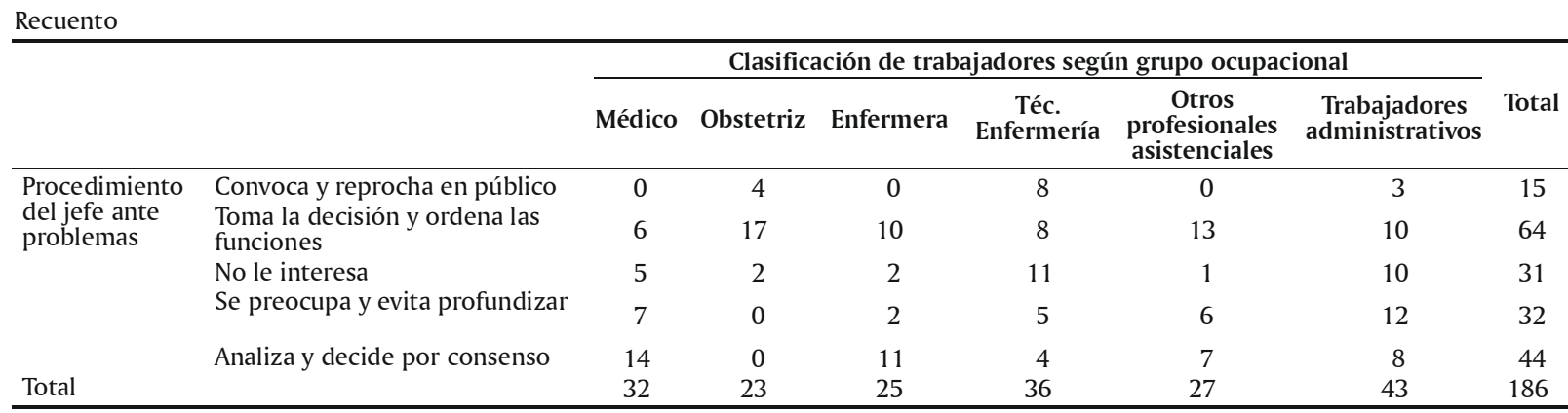

\begin{tabular}{lccc}
\multicolumn{4}{c}{ Pruebas de chi-cuadrado } \\
\hline & Valor & gl & $\begin{array}{c}\text { Sig. asintótica } \\
\text { (bilateral) }\end{array}$ \\
\hline Chi-cuadrado de Pearson & $73.104^{\text {a }}$ & 20 & .000 \\
Razón de verosimilitud & 83.032 & 20 & .000 \\
$\begin{array}{l}\text { Asociación lineal por } \\
\text { lineal }\end{array}$ & .007 & 1 & .935 \\
Número de casos válidos & 186 & & \\
\hline a. & & &
\end{tabular}

a. 12 casillas $(40.0 \%)$ tienen una frecuencia esperada inferior a

5. La frecuencia mínima esperada es 1.85 . 


\section{Estadística de prueba experimental}

$$
\chi^{2}=\frac{(|a d-b c|-0.5 n)^{2} n}{(a+c)(b+d)(a+b)(c+d)}=8.1035
$$

El valor que alcanza chi-cuadrado experimental es superior al valor tabular, lo que permite que la hipótesis nula sea rechazada a un nivel de significancia de 5\%; por lo que se concluye que los procedimientos adoptados por el jefe ante el surgimiento de problemas influyen positivamente en la opinión que tienen al respecto los trabajadores, según el grupo ocupacional al que pertenecen.

Facultación al trabajador de autonomía para tomar decisiones frente al tipo de trabajadores según funciones asignadas

\section{Hipótesis 1:}

$\mathbf{H}_{\mathrm{o}}$ : La importancia de la autonomía del personal para tomar decisiones es independiente a la opinión que tienen los trabajadores de la institución.

$\mathbf{H}_{1}$ : La importancia de la autonomía del personal para tomar decisiones influye positivamente en la opinión que tienen los trabajadores de la institución.

Los datos han sido clasificados en una tabla de contingencia para probar la hipótesis de independencia de criterios. Se ha considerado un nivel de significancia $\mathbf{a}=\mathbf{0 . 0 5}$ y 1 grados de libertad cuyo valor tabular es de $c^{2}{ }_{(0.05,1)}=3.8416$, que luego será comparado con un chi-cuadrado experimental para la aceptación o rechazo de la hipótesis nula.

\section{Estadística de prueba experimental}

$$
\chi^{2}=\frac{(|a d-b c|-0.5 n)^{2} n}{(a+c)(b+d)(a+b)(c+d)}=0.4852
$$

El valor que alcanza chi-cuadrado experimental es inferior al valor tabular lo que permite que la hipótesis alterna sea rechazada a un nivel de significancia de $5 \%$; por lo que se concluye que la importancia de la autonomía del personal para tomar decisiones es independiente a su status de pertenecer a un grupo ocupacional específico.

\section{Nivel de percepción de las pacientes}

Frente a la pregunta de los Hospitales e Institutos que recuerda (Cuadro 6), los resultados ubican al IEMP en el tercer lugar con un $12 \%$.

La explicación de esta ubicación va por el lado de que los dos hospitales mencionados en primer y segundo lugar (Dos de Mayo y Loayza, respectivamente), son hospitales generales en tanto que el IEMP es un centro especializado. El resultado obtenido en esta pregunta también

Cuadro 5. Autonomía del personal para tomar decisiones vs. Tipo de trabajadores según funciones asignadas.

Recuento

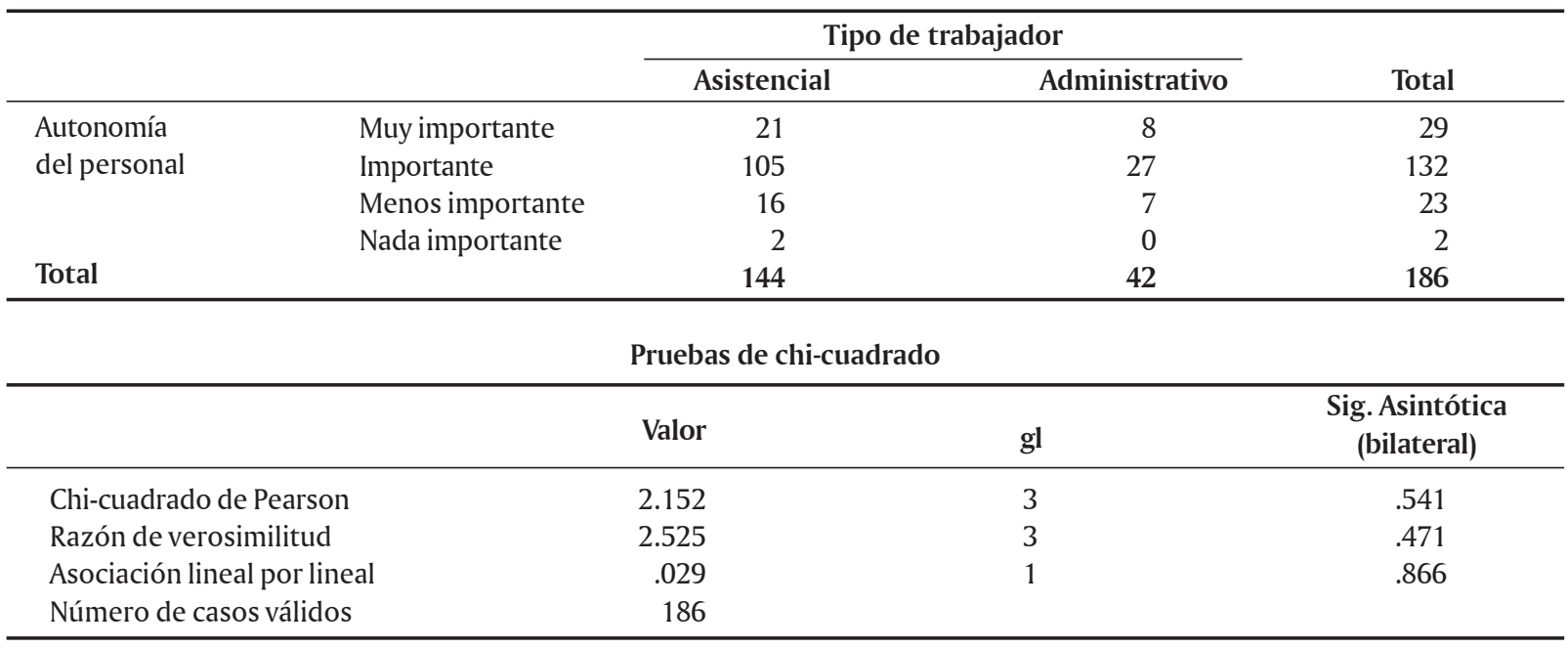

a. 2 casillas $(25.0 \%)$ tienen una frecuencia esperada inferior a 5. La frecuencia mínima esperada es .45 . 
destaca el hecho de que en la primera mención resalten todos los hospitales que brindan atención gineco-obstétrica lo que no ocurre en las menciones múltiples, por cuanto en ella también figuran otros centros asistenciales que tienen misiones diversos (ejemplo, Hospital del Niño, Instituto Mogrovejo, etc.), que también desempeñan roles muy importantes para la salud de la sociedad peruana.

Cuadro 6. nivel de recordación de los hospitales e institutos generales del Ministerio de Salud -Primera mención-

\begin{tabular}{clcc}
\hline \multirow{2}{*}{ Válido } & $\begin{array}{c}\text { Válido } \\
\text { (\%) }\end{array}$ & $\begin{array}{c}\text { Acumulado } \\
\text { (\%) }\end{array}$ \\
\hline \multirow{2}{*}{ Dos de Mayo } & 36.0 & 36.0 \\
Arzobispo Loayza & 18.0 & 54.0 \\
Maternidad de Lima & 12.0 & 66.0 \\
Cayetano Heredia & 8.0 & 74.0 \\
Hipólito Unanue & 6.0 & 80.0 \\
María Auxiliadora & 6.0 & 86.0 \\
San Bartolomé & 4.0 & 90.0 \\
Otros hospitales & 10.0 & 100.0 \\
Total & $\mathbf{1 0 0 . 0}$ & \\
\hline
\end{tabular}

La frecuencia de visitas (Gráfico 8) que realizan las pacientes "clientes" a la Maternidad de Lima para atenderse varía; así tenemos visitas semanales, quincenales, mensuales, bimensuales, trimestrales, semestrales, etc. Las visitas mensuales ocupan el primer lugar con un $24 \%$. Con un $20 \%$ figuran aquellas que realizan visitas quincenalmente, seguida de aquellas que manifiestan que lo hacen cada vez que se embarazan (18\%). Las que lo hacen trimestralmente figuran con un $14 \%$, en tanto que los que realizan sema-

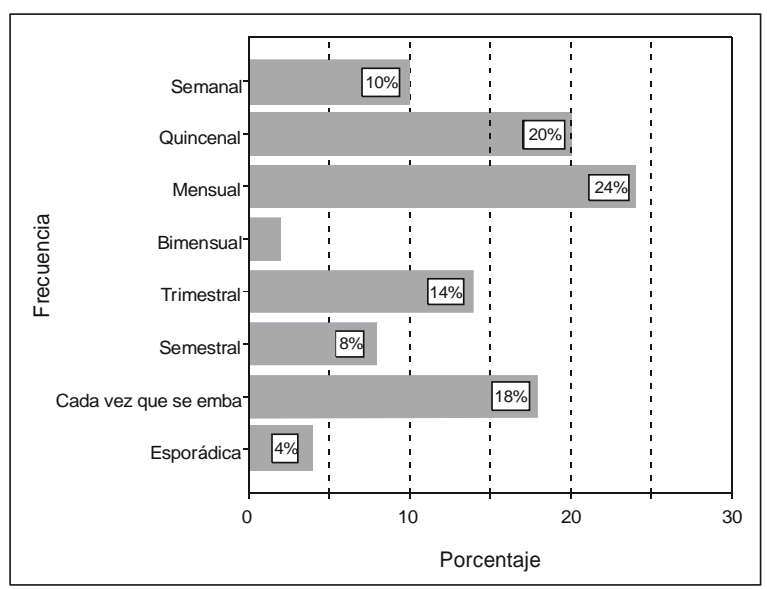

Gráfico 8. Frecuencia de visita a la Maternidad de Lima. nalmente ascienden al $10 \%$, seguida de aquellas que visitan semestralmente la institución con un $8 \%$.

La presencia masiva de "clientes" mujeres encuestadas y la frecuencia de visitas para recibir atenciones especializadas corroboran el carácter altamente especializado en Gineco-Obstetricia del Instituto Especializado Materno Perinatal.

En relación a su nivel de aceptación de los servicios que oferta el IEMP (Gráfico 9), un 74\% de las pacientes "clientes" califican positivamente al nivel de atención recibida en este nosocomio (4\% Muy buena y 70\% Buena). Ello significa que el IEMP goza de una buena performance a nivel de este segmento de usuarias de la Gran Lima. Como contraparte, los $26 \%$ de las pacientes «clientes» distribuidas en $20 \%$ regular y $6 \%$ mala, muestran su insatisfacción con el nivel de atención recibida.

Al preguntársele a los que manifestaron su aprobación de la calidad de servicios, si estarían dispuestos a recomendar a sus familiares y amigos para que se atiendan en este hospital, el $74 \%$ se mostraron dispuestos a recomendar a sus allegados, mientras que para un $26 \%$ restante de la muestra la idea de hacer tal recomendación no se encuentra dentro de sus planes inmediatos.

De la misma forma, en el Gráfico 10 se presentan las recomendaciones de las pacientes que manifestaron su insatisfacción con la calidad de los servicios que oferta el IEMP.

El 46\% manifiestan que deben mejorar el trato hacia las pacientes "clientes", seguido de un $31 \%$ que sugieren que deben agilizar en la

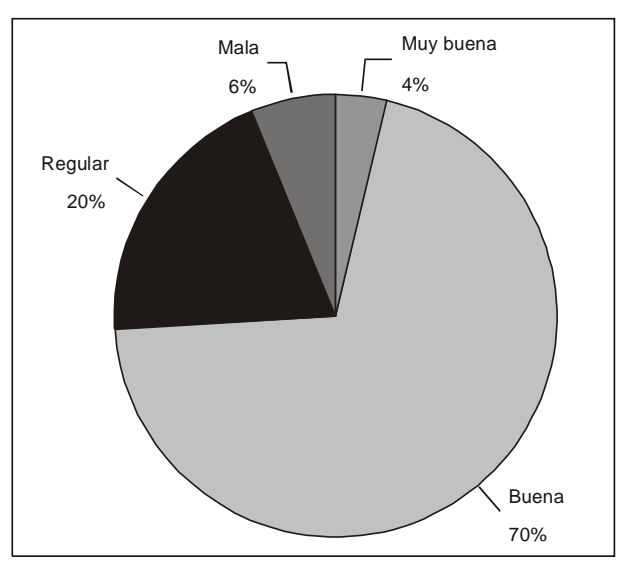

Gráfico 9. Calificación del Nivel de atención en el IEMP. 


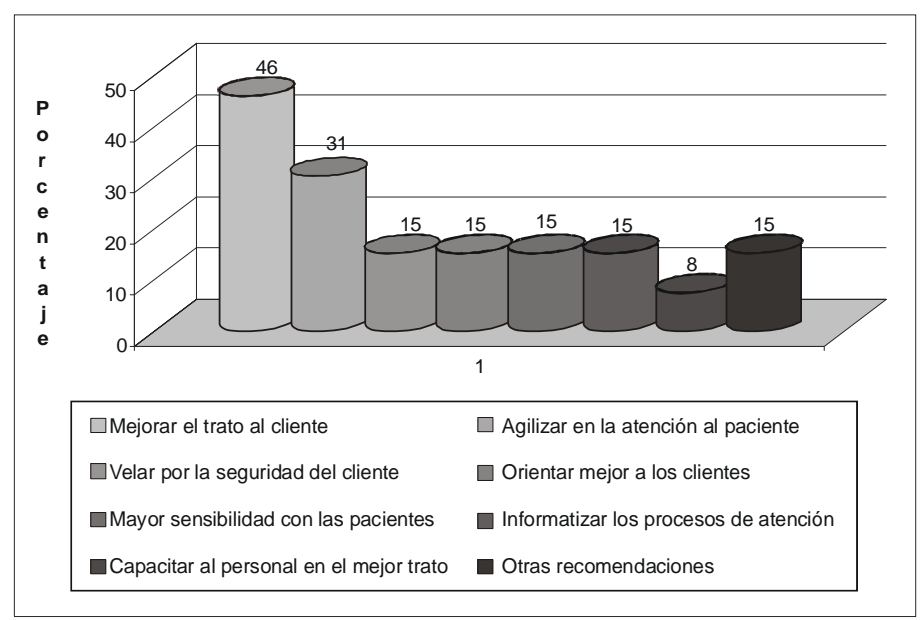

Gráfico 10. Recomendaciones de pacientes "clientes" disconformes a nivel de atencion del IEMP.

atención al paciente. Las otras recomendaciones tales como velar por la seguridad del cliente, mayor sensibilidad con las pacientes, orientar mejor a las pacientes e informatizar los procesos de atención, se encuentran empatados con un $15 \%$, ocupando el tercer lugar. Adicional a otras recomendaciones, la necesidad de capacitar al personal en el mejor trato a las pacientes también figura con un $8 \%$.

A partir de tales resultados es interesante analizar que la mayoría de las recomendaciones van por el lado de mejora de relaciones entre colaboradores- pacientes. Ya se comienzan a visualizar las necesidades de capacitación al personal en temas relacionados al proceso de sensibilización en las relaciones humanas.

En relación a la calificación de las pacientes al trabajo que realizan los diferentes grupos ocupacionales, los encuestados se inclinan en opinar que la atención que brinda el médico es la mejor con respecto a los demás profesionales, y así lo demuestra un $96 \%$ de las preferencias; por otro lado, la atención de la que son objeto por parte de las obstetrices se encuentra relegado a un segundo lugar con un 68\% de las preferencias de las pacientes, para finalmente y por no ser menos importante las enfermeras también gozan de esta preferencia compartiendo un razonable $60 \%$, que no se encuentra muy lejos del resultados obtenido por sus colegas obstetriz.

En vista de tales resultados podemos deducir que el médico es el profesional cuyo desempeño las pacientes califican como muy bueno y bueno, en relación a las obstetrices y enfermeras. Sin embargo, el nivel de aprobación tan- to de obstetrices y enfermeras sobrepasa el 50\%, lo cual demuestra que también sus desempeños son buenos.

Los otros profesionales, tales como el tecnólogo médico, obtiene el $34 \%$ de calificación buena, el $66 \%$ de regular y $2 \%$ de calificación mala; en tanto que para el psicólogo el 28\% dicen que es buena y el $72 \%$ manifiestan que son regulares y malas. De la misma forma, el 30\% considera que las asistentas sociales brindan buenos servicios, el $70 \%$ dicen que son regulares, malos y muy malos. Con tales resultados queda demostrado que las dos terceras partes de los encuestados opina que los trabajos de tecnólogos médicos, psicólogos y asistentas sociales son regulares y malos, en tanto que el tercio restante manifiestan que sus trabajos son buenos y muy buenos.

Los resultados del grupo de profesionales técnicos muestran calificaciones desiguales. Así mientras que a los técnicos de laboratorio el $82 \%$ califica su desempeño como bueno y muy bueno, el $64 \%$ dice que el trabajo de los técnicos de enfermería es bueno, y solo el 34\% de los encuestados manifiestan que el trabajo de los técnicos de nutrición es bueno. Visto así, las pacientes reconocen y valoran como mejores desempeños primero, los trabajos de los técnicos de laboratorio, segundo los desempeños de los técnicos de enfermería y en tercer lugar los trabajos de los técnicos de nutrición. Esta tendencia refleja la necesidad de ir perfeccionando las tareas para obtener una aceptación marcada por el lado de los que consideren como muy buena y buena. 


\section{A MODO DE CONCLUSIÓN}

Las siguientes, son a nuestro juicio, las conclusiones a las cuales arribamos con la presente investigación:

1. Los resultados revelan que la capacitación permanente al personal viene a ser la estrategia más eficaz que deberían utilizar los centros asistenciales del Ministerio de Salud para motivar a sus trabajadores. En ese orden de ideas, la promoción y ascensos de personal sobresale como la segunda estrategia a utilizar para motivar. El reconocimiento público que debe realizar la institución al trabajador ocupa un lugar expectante, el mismo que es concordante con el buen trato que deberían dar sus jefes. De la misma forma, los trabajadores reclaman contar con equipos modernos y materiales en calidad y cantidades oportunas para que desempeñen mejor su trabajo. Las estrategias económicas, referidas a los bonos por alimentos y los incentivos por productividad, ocupan el tercer grupo de preferencias para motivar. Con tales resultados, podemos concluir que la jerarquía de los estímulos organizacionales en el Sector Salud -vía investigación en el IEMP- muestra una relación inversa a la propuesta por Abraham Maslow, en su famosa teoría de jerarquía de necesidades. Ello implica, que las mejores estrategias de gestión en los organismos asistenciales del Sector Salud deben estar dirigidas a satisfacer, en primer lugar necesidades de capacitación, promoción y ascensos, y reconocimiento del trabajador -propios de necesidades de orden social y psicológico-, en segundo lugar, mejora de condiciones laborales, vía equipamiento permanente y materiales adecuados para su trabajo; y en tercer lugar los estímulos económicos.

2. En relación al beneficio proporcionado directamente al trabajador en los últimos 12 meses, revela que el buen trato de sus jefes ocupa el primer lugar con un $20.4 \%$, seguido de los uniformes y equipos facilitados para su trabajo, con un $11.8 \%$. La capacitación laboral tiene el $10.2 \%$, seguido por los beneficios pecuniarios como mejora de los bonos por alimentos $(3.8 \%)$ y incremento del incentivo por productividad (3.2\%). Sin embargo, un representativo $42 \%$ de los entrevistados manifiestan no haberse beneficiado con ningu- na acción estimuladora. Tales resultados revelan que el Instituto Especializado Materno Perinatal, ha priorizado -a excepción del buen trato, que no necesariamente puede ser un estímulo y la capacitación- los aspectos materiales y pecuniarios como estrategias de gestión organizacional. Contrastando tales resultados con los obtenidos en el Cuadro $\mathrm{N}^{\circ}$ 1 podemos concluir que el IEMP viene utilizando estrategias que no necesariamente estimulan a su personal para que sea productivo, y por ende mejorar la calidad de los servicios e imagen institucional. Ello independientemente de la afirmación de casi el $50 \%$ de los trabajadores que manifiestan no haber sido estimulados en los últimos doce meses.

3. Los resultados del Cuadro 3 muestran que en el IEMP coexisten tres estilos de gestión practicada por los directivos y jefes intermedios: el primero, que se caracteriza por ser autoritario y dominante (42.5\%), el segundo que es indiferente a la realidad de la unidad o área de trabajo (16.7\%), y el tercero que escucha a los trabajadores y toma decisiones por consenso $(40.9 \%)$. Este resultado, al cruzar con la información referida a la calificación del trato que reciben de su jefe, se descubre que los que califican como muy bueno y bueno tienen por jefes a aquellos que practican estilos democráticos y participativos; en tanto que aquellos que califican el trato como regular y malo tienen como jefes a aquellos que practican estilos autoritarios y dominantes. Pero ello no es todo: al cruzar los estilos de gestión con los trabajadores clasificados según grupos ocupacionales (Cuadro 4), vemos que los estilos autoritario y dominantes son mas acentuados en las Obstetrices, seguido de los trabajadores administrativos y técnicos de enfermería; en tanto que los estilos democráticos y participativos son mas acentuados en los médicos, seguido de las enfermeras. De acuerdo con tales resultados podemos concluir que los estilos jefaturales caracterizados por ser democráticos y participativos tienen una alta percepción, alcanzando al $41 \%$ de los trabajadores, así como los que perciben que sus jefes tienen estilos autoritarios y dominantes alcanzan el $42.5 \%$ de los trabajadores. Asimismo debemos concluir que los estilos democráticos y participativos son más acentuados en los 
médicos y enfermeras, en tanto que los estilos autoritarios y dominantes son más acentuados en las Obstetrices, trabajadores administrativos y técnicos de enfermería.

4. Los resultados revelan que los trabajadores del Sector Salud -vía investigación en el IEMP- requieren tener autonomía para tomar decisiones en actividades inherentes a su función, dentro de las normas institucionales y los objetivos de la organización, para realizar a satisfacción su trabajo. Para ello los directivos y jefes intermedios deben aprender a confiar y delegar las funciones en sus colaboradores. Al respecto, el Gráfico 4 muestra que contar con autonomía para tomar decisiones -el 16\% consideran que es muy importante y el $71 \%$, dicen que es importantees determinante para su desempeño laboral. Esta aseveración es corroborada cuando los encuestados manifiestan que la delegación de funciones en los trabajadores es muy importante $(17 \%)$ e importante $(70 \%)$. Con tales resultados podemos concluir que una dirección institucional democrática y participativa, caracterizada por la confianza en sus trabajadores para que gocen de facultades y tomen decisiones en las actividades inherentes a sus funciones, es la estrategia más indicada para desarrollar las organizaciones.

\section{Notas}

1 Esta nueva denominación acerca de los trabajadores, no solo refleja la modificación de argots o terminologías administrativas, sino fundamentalmente un cambio de actitud de los líderes organizacionales de vanguardia, quienes actualmente conciben a los trabajadores como activos que tienen conocimientos, capacidades y actitudes, a través de los cuales la organización genera valor. De expresiones habituales de antaño refiriéndose al personal como subordinados, fuerza de trabajo, subalternos o trabajadores, ahora hemos pasado a referirnos a ellos como colaboradores, clientes internos, etc. De la misma forma, de considerar al personal como recurso humano ahora se prefiere catalogarlos como capital humano y/o capital intelectual, denominaciones que utilizaremos indistintamente para referirnos a los trabajadores que colaboran con las organizaciones.

2 Una posición de vanguardia referida a las diversas funciones en la organización, relacionadas con la incorporación, mantenimiento y desarrollo, así como la exclusión del personal, van por el lado de concebirlas como Gerencia del Potencial Humano, Gestión del Talento Humano o Gestión del Capital Humano.

\section{BIBLIOGRAFÍA}

Alles, Martha (2004). Dirección Estratégica de los Recursos Humanos. Quinta edición. Buenos Aires, Granica Ediciones, 475 p.

Blanchard, Ken (1996). Empowerment. Bogotá, Editorial Norma, 176 p.

Borras, Mateo. El nuevo rol de los trabajadores a través de la visión del directivo de recursos humanos. En: Alta Dirección. Barcelona, Edición № 208, Noviembre/ Diciembre 1999, $480 \mathrm{p}$.

Brito P. y Lazo, O. El trabajo médico en el Perú. Lima, Organización Panamericana de la Salud - Universidad Peruana Cayetano Heredia, 1990.

Chiavenato, Adalberto (2003). Gestión del talento humano. Bogotá, Mc Graw-Hill, 475 p.

Drucker, Peter (1999). Los desafíos de la gerencia para el Siglo XXI. Bogotá, Editorial Norma, 277 p.

Drucker, Peter (2002). La gerencia en la sociedad futura. Santa Fe de Bogotá, Editorial Norma, 290 p.

Drucker, Peter. "La productividad del trabajador del conocimiento: Máximo desafío”. En: Harvard Deusto Business Review. Madrid, Edición 98, Oct. 2000.

Ernst \& Young. "Gestión por competencias, innovación en la gestión empresarial", En: Cuadernos Cinco Días. Madrid, Fascículo № 6, 1998.

Flores García, Javier (2001). El comportamiento humano en las organizaciones. Lima, Universidad del Pacífico, 536 p.

Gonzales, Luis. "La Dirección de Recursos Humanos en el horizonte del 2000". En: Alta Dirección. Barcelona, Edición 204, 1999.

Méndez Alvarez, Carlos (2001). Metodología. Bogotá, Edit. Mc Graw-Hill, 246 p.

Ministerio de Salud / Instituto de Desarrollo de Recursos Humanos. Políticas de Desarrollo de Recursos Humanos de Salud. Lima, 2004, $25 \mathrm{p}$.

Ministerio de Salud. Lineamientos de Política Sectorial para el Período 2002-2012 y Principios Fundamentales para el Plan Estratégico 
Sectorial del Quinquenio Agosto 2001- Julio 2006. Lima, 2002, 57 p.

Ministerio de Salud. Proyecto generación de capacidades. Análisis Sectorial de los Recursos Humanos en Salud. Bases para los Lineamientos de Política de Salud. Lima, 1998.

Organización Internacional del Trabajo (OIT) / Oficina Regional para América Latina y el Caribe. Panorama Laboral 2005. Lima, 2005, $118 \mathrm{p}$.

Organización Internacional del Trabajo (OIT). Relación de Trabajo, Informe V (1). Ginebra, 2005, 92 p.

Organización Panamericana de la Salud / Organización Mundial de la Salud. Motivación de los trabajadores de la salud y reforma del sector salud. Montgomery, 2001.

RICSE, Carlos (2000). Análisis de Información de Recursos Humanos en Salud. Lima, Organización Panamericana de la Salud, 38 p.

Robbins, Stephen (1999). Comportamiento organizacional. Octava edición. México D.F., Prentice May, 816 p.

Robbins, Stephen y De Cenzo, David (1996). La Dirección del Recurso Humano. Nueva Cork, Addison Wesley.

Sánchez-Runde, Carlos. Tres modelos de estrategia y dirección de recursos humanos. En: Harvard Deusto Business Review. Madrid, Edición 113, 2003. 